\title{
NEW TAXA AND NEW COMBINATIONS IN JUNGERMANNIALES
} (HEPATICAE)

\section{НОВЫЕ ТАКСОНЫ И НОВЫЕ КОМБИНАЦИИ ПЕЧЕНОЧНИКОВ ИЗ ПОРЯДКА ЈUNGERMANNIALES (HEPATICAE)}

\author{
N.A.KONSTANTINOVA ${ }^{1} \&$ A.A.VILNET ${ }^{1}$ \\ Н.А.КОНСТАНТИНОВА ${ }^{1}$, А.А. ВИЛЬНЕТ ${ }^{1}$
}

\begin{abstract}
The names of one new family (Hygrobiellaceae (Jørg.) Konstant. \& Vilnet) and 7 new genera (Pseudolophozia Konstant. \& Vilnet, Pseudotritomaria Konstant. \& Vilnet, Schljakovia Konstant. \& Vilnet, Schljakovianthus Konstant. \& Vilnet, Lophoziopsis Konstant. \& Vilnet, Biantheridion (Grolle) Konstant. \& Vilnet, Heterogemma (Jørg.) Konstant. \& Vilnet) are validated. Correspondingly, 21 new combinations are made in these genera.
\end{abstract}

Резюме

Обнародованы названия одного нового семейства (Hygrobiellaceae (Jørg.) Konstant. \& Vilnet) и 7 новых родов (Pseudolophozia Konstant. \& Vilnet, Pseudotritomaria Konstant. \& Vilnet, Schljakovia Konstant. \& Vilnet, Schljakovianthus Konstant. \& Vilnet, Lophoziopsis Konstant. \& Vilnet, Biantheridion (Grolle) Konstant. \& Vilnet, Heterogemma (Jørg.) Konstant. \& Vilnet), а также 21 новая комбинация в этих родах.

KEYWORDS: liverworts, taxonomy, nomenclature, new taxa

The last ten years are characterized by a breathtaking speed of development of molecular study of hepatics. As a result, the ideas about phylogeny of hepatics have changed drastically (Davis et al., 2004; Yatsentyuk et al.,2004; HeNygren et al.,2006; Forrest et al., 2006; Hentschel et al., 2007; De Roo et al. ,2007; Crandal-Stotler et al.,2008; Vilnet et al., 2008, 2009). Many genera traditionally accepted as a "good" ones were found to be polyphyletic, which forced to search for new taxonomic solutions for their placement. Our study of Jungermanniales (Vilnet et al., 2008, 2009, unpublished data) is one those, that demonstrate a high level of incongruence between traditional taxonomy and novel ideas of their relationships. This require a certain rearrangement of taxa, a number of new combinations and description of several new genera and one new family for such recently found groups. These names are needed for the simultaneously published checklist of liverworts of Russia (Konstantinova, Bakalin et al., 2009).

\section{NEW FAMILY}

Hygrobiellaceae (Jørg.) Konstant. \& Vilnet, stat. nov. - Based on Cephaloziaceae Mig. subfam. Hygrobielloideae (Jørg.) R.M.Schust. ex Grolle, J. Bryol. 7: 210. 1972.

The family includes one genus, Hygrobiella Spruce.

\section{NEW GENERA}

Pseudolophozia Konstant. \& Vilnet, gen.nov. - Genus hoc generi Lophoziae s. str. affine sed foliis trilobatis frequenter alatis, cellulis caulium saepiuscule homogeneis in sectione transversali, gemmis rubello-fuscis vel ferrugineis et ITS1-2 $n r D N A$ et trnL-F cpDNA sequentiae differt.

1 - Polar-Alpine Botanical Garden, Kola Sci. Center of Russian academy of Scienses, Kirovsk-6, Murmansk Province 184256 Russia - Россия 184256, Кировск-6 Мурманской области, Полярно-альпийский ботанический сад-институт КНЦ РАН; e-mail: nadya50@mail.ru \& anya_v@list.ru 
Typus: Pseudolophozia sudetica (Nees ex Huebener) Konstant. \& Vilnet. (Jungermannia sudetica Nees ex Huebener).

Species:Pseudolophozia sudetica (Nees ex Huebener) Konstant. \& Vilnet, comb. nov. Jungermannia sudetica Nees ex Huebener, Hepaticol. Germ. 142 Mannheim. 1834.

Pseudolophozia sudetica var. anomala (Schljakov) Konstant. \& Vilnet, comb. nov. - Lophozia alpestris (F.Weber) Evans var. anomala Schljakov, Novosti Syst. Nizљ. Rast. 11: 352. 1974.

Pseudolophozia debiliformis (R.M.Schust. \& Damsh.) Konstant. \& Vilnet, comb. nov. - Lophozia debiliformis R.M.Schust. \& Damsh., Phytologia 63(5):326. 1987.

Pseudolophozia debiliformis var. concolor (R.M.Schust. \& Damsh.) Konstant. \& Vilnet, comb. nov. - Lophozia debiliformis var. concolor R.M.Schust. \& Damsh., Phytologia 63: 326. 1987.

Pseudotritomaria Konstant. \& Vilnet, gen. nov. - Planta minima, 1.0-1.4(-1.6) mm lata, foliis subsymmetricis, trilobatis,parietibus cellularum in angulis perincrassatis. Genus hoc genera Tritomaria et Saccobasis appropinquat sed ab eis marginibus foliorum perichaetialium undulatocrispatis, spinuloso-dentatis vel spinuloso-ciliatis et ITS1-2 nrDNA et trnL-F cpDNA sequentia differt.

Typus: Pseudotritomaria heterophylla (R.M.Schust.) Konstant. \& Vilnet (Tritomaria heterophylla R.M.Schust.).

Pseudotritomaria heterophylla (R.M.Schust.) Konstant. \& Vilnet, comb. nov. - Tritomaria heterophylla R.M.Schust., Canad. J. Bot. 36: 172. 1958.

Schljakovia Konstant. \& Vilnet, gen.nov. Genus hoc generi Orthocauli simile sed foliis profunde bilobatis, caulibus gemmiferis haud modificatis et ITS1-2 nrDNA et trnL-F cpDNA sequentia recedit.

Typus: Schljakovia kunzeana Konstant. \& Vilnet (Jungermannia kunzeana Huebener).

Schljakovia kunzeana Konstant. \& Vilnet, comb. nov. - Jungermannia kunzeana Huebener Hepaticol. Germ.: 115. 1834.

Schljakovianthus Konstant. \& Vilnet, gen. nov. - Genus hoc generi Orthocauli simile sed foliis quadrilobatis ad 0.6 laminae partitis, marginibus sinuum distincte revolutis, cuticula perpapillosa, gemmis nullis et ITS1-2 nrDNA et trnL$F$ cpDNA sequentia differt.

Typus: Schljakovianthus quadrilobus (Lindb.) Konstant. \& Vilnet (Jungermannia quadriloba Lindb.).

Schljakovianthus quadrilobus (Lindb.) Konstant. \& Vilnet, comb. nov. - Jungermannia quadriloba Lindb., Meddeland Soc. Fauna Fl. Fenn. 9: 162, 1889 (nom. subnud.) et in Arnell \& Lindb., Kongl. Svenska Vetenskapsakad. Hand1. 23 (5): 55. 1889.

Schljakovianthus quadrilobus var. glareosa (Jwrg.) Konstant. \& Vilnet, comb. nov. - Jungermannia quadriloba var. glareosa Jwrg., Bergens Mus. Erbok 18. s. p. 1895.

Schljakovianthus quadrilobus var. collenchymatica (R.M. Schust.) Konstant. \& Vilnet, comb. nov. - Lophozia quadriloba (Lindb.) A.Evans var. collenchymatica R.M. Schust. Hepat. Anthocer. N. Amer. 2: 281. 1969.

Lophoziopsis Konstant. \& Vilnet, gen. nov. - Genus hoc generi Lophoziae s. str. simile sed ab eo gemmis rubris ad purpurascentibus vel rubello-fuscis, cellulis leptodermaticis, bracteis femineis saepe dentatis et ITS1-2 nrDNA et trnLF cpDNA sequentia recedit.

Typus Lophoziopsis excisa (Dicks.) Konstant. \& Vilnet (Jungermannia excisa Dicks.). Lophoziopsis excisa (Dicks.) Konstant. \& Vilnet, comb. nov. - Jungermannia excisa Dicks., Pl. Crypt. Brit. Fasc. 3: 11. 1793.

Lophoziopsis excisa var. elegans (R.M. Schust.) Konstant. \& Vilnet, comb. nov. - Lophozia excisa (Dicks.) Dumort. var. elegans R.M. Schust., Hepat. Anthocer. N. Amer. 2: 522. 1969.

Lophoziopsis excisa var. infuscata (R.M. Schust. \& Damsh.) Konstant. \& Vilnet, comb. nov. - Lophozia excisa (Dicks.) Dumort. var. infuscata R.M. Schust. \& Damsh., Meddel. Gr?nland 199: 94. 1974.

Lophoziopsis excisa var. succulenta (R.M. Schust. \& Damsh.) Konstant. \& Vilnet, comb. nov. - Lophozia excisa (Dicks.) Dumort. var. succulenta R.M. Schust. \& Damsh., Meddel. Gr?nland 199: 96. 1974.

Lophoziopsis longidens (Lindb.) Konstant. \& Vilnet, comb. nov. - Jungermannia longidens 
Lindb., Helsigfors Dagblad 1876 (323): 2. 1876. et Bot. Not. 27. 1877.

Lophoziopsis pellucida (R.M.Schust.) Konstant. \& Vilnet, comb. nov. - Lophozia pellucida R.M.Schust., Canad. J. Bot. 39: 978. 1961.

Lophoziopsis perssonii (H.Buch \& S.W.Arnell) Konstant. \& Vilnet, comb. nov. Lophozia perssonii H.Buch \& S.W.Arnell in Buch, Bot. Not. 1944: 382, figs. 1-2. 1944.

Lophoziopsis polaris (R.M.Schust.) Konstant. \& Vilnet, comb. nov. - Lophozia alpestris (Schleich ex F. Weber) A.Evans ssp. polaris R.M. Schust., Hepat. Anthocer. N. Amer. 2: 614, NewYork, 1969.

Lophoziopsis polaris var. sphagnorum (R.M.Schust.) Konstant. \& Vilnet, comb. nov. Lophozia alpestris (Schleich ex F. Weber) A.Evans ssp. polaris R.M. Schust. f. sphagnorum R.M.Schust., Hepat. Anthocer. N. Amer. 2: 619, fig. 218: 15-19. 1969.

Lophoziopsis propagulifera (Gottsche) Konstant. \& Vilnet, comb. nov. - Jungermannia propagulifera Gottsche, Int. Polarforsch., Deutsch. Exped. 2: 451, 1890.

Lophoziopsis rubrigemma (R.M.Schust.) Konstant. \& Vilnet, comb. nov. - Lophozia rubrigemma R.M.Schust., Hepat. Anthocer. N. Amer. 2: 621. 1969.

Biantheridion (Grolle) Konstant. \& Vilnet, stat. nov. - Jamesoniella sect. Biantheridion Grolle, Trans. Brit. Bryol. Soc. 4, 4: 662.1964

Biantheridion undulifolium (Nees) Konstant. \& Vilnet, comb. nov. - Jungermannia schraderi var. undulifolia Nees, Naturgesch. Eur. Leberm. 1: $306,1833$.

Heterogemma (Jørg.) Konstant. \& Vilnet, stat. nov. - Lophozia sect. Heterogemma Jørg., Bergens Mus. Skr. 16: 1959, 1934.

Heterogemma laxa (Lindb.) Konstant. \& Vilnet, comb. nov. - Jungermannia laxa Lindb., Acta Soc. Sci. Fenn. 10: 539. 1875.

Heterogemma capitata (Hook.) Konstant. \& Vilnet, comb. nov. - Jungermannia capitata Hook., Brit. Jungermann.: Tab. 80. 1816.

\section{ACKNOLEDGEMENTS}

We thank M.S. Ignatov, J. Váňa, L. Söderström, A. Hagborg for numerous comments and suggestions. Dr. R. Ochyra is cordially thanked for trans- lation of Latin diagnoses. This work was partly supported by RFBR 09-04-00281, 06-05-64137.

\section{LITERATURE CITED}

CRANDAL-STOTLER, B.J., R.E. STOTLER \& D.G. LONG 2008. Morphology and classification of the Marchantiophyta. - Goffinet, B. \& A.J.Shaw (eds.) Bryophyte Biology, 2 ed. Cambridge: $1-54$.

DAVIS, E.C. 2004. A molecular phylogeny of leafy liverworts (Jungermanniidae: Marchantiophyta). - Monogr. Syst. Bot. Missouri Bot. Garden 98: 61-86.

DE ROO, R.T., T.A. HEDDERSON \& L. SÖDERSTRÖM 2007. Molecular insights into the phylogeny of the leafy liverwort family Lophoziaceae Cavers. - Taxon 56: 301-314.

FORREST, L.L., E.C.DAVIS, D.G.LONG, B.J. CRANDALSTOTLER, A. CLARK \& M.L. HOLLINGSWORTH 2006. Unravelling the evolutionary history of the liverworts (Marchantiophyta): multiple taxa, genomes and analyses. Bryologist 109: 303-334.

HENTSCHEL, J., J.A. PATON, H. SCHNEIDER \& J. HEINRICHS 2007. Acceptance of Liochlaena Nees and Solenostoma Mitt., the systematic position of Eremonotus Pearson and notes on Jungermannia L. s.l. (Jungermanniidae) based on chloroplast DNA sequence data. - Pl. Syst. Evol. 268 : 147-157.

HE-NYGREN X., A. JUSLÉN, D.,GLENNY \& S. PIIPPO 2006. Illuminating the evolutionary history of liverworts (Marchantiophyta) - towards a natural classification. - Cladistics 22: 1-31.

KONSTANTINOVA, N.A., V.A. BAKALIN ET AL. 2009. Checklist of liverworts (Marchaniophyta) of Russia. - Arctoa 18: 1-66.

[VILNET, A.A., N.A. KONSTANTINOVA \& A.V. TROITSКY] ВИЛЬНЕТ А.А., Н.А. КОНСТАНТИНОВА, А.В. ТРОИЦКИЙ 2007. К молекулярной филогении семейства Gymnomitriaceae H.Klinggr. (Hepaticae). - [On molecular phylogeny of Gymnomitriaceae H.Klinggr. (Hepatiсае) ] Мат. международн. конф. «Вычислительная филогенетика и геносистематика «ВФГС»» КМК, Москва [Proceedings of Conference "Compulational phylogenetics and molecular systematics "COMS'2007", KMK, Moscow]: 24-26.

VILNET, A.A., N.A. KONSTANTINOVA \& A.V. TROITSKY 2008. Phylogeny and systematics of the genus Lophozia s. str. (Dumort.) Dumort. (Hepaticae) and related taxa from nuclear ITS1-2 and chloroplast trnL-F sequences. - Mol. Phylogenet. Evol. 47: 403-418.

[VILNET, A.A., N.A. KONSTANTINOVA \& A.V. TROITSKY] ВИЛЬНЕТ А.А., Н.А. КОНСТАНТИНОВА, А.В. ТРОИЦКИЙ 2009. Геносистематика и новый взгляд на филогению и систему печеночников. - [Genosystematics and new insight on phylogeny and taxonomy of liverworts] Молек. биол. [Molec. Biol.] 43 (5): 1-12.

YATSENTYUK, S.P., N.A. KONSTANTINOVA, M.S. IGNATOV, J. HYVÖNEN \& A.V. TROITSKY 2004. On phylogeny of Lophoziaceae and related families (Hepaticae, Jungermanniales) based on trnL-trnF intron-spacer sequences of chloroplast DNA. - Monographs in Systematic Botany from the Missouri Botanical Garden 98: 150-167. 